\title{
Features of the kinetics of mass transfer processes in the process of firing ceramic materials
}

\author{
O.M. Nedbailo (0000-0003-1416-9651), O.G. Chernyshyn (0000-0001-7702-894X) \\ The State Enterprise "Engineering Center "Drying"” Institute of Engineering Thermophysics of NAS of Ukraine, \\ str. Bulakhovskogo, 2, Bldg. 2, Kyiv, 03164, Ukraine \\ Tel.: +380444240279, tel./fax +380444243283 \\ E-mail:icsushka@gmail.com
}

Article info: received 17.11.2018, revised 03.12.2018, accepted 21.12.2018

Nedbailo, O.M., Chernyshyn, O.G. (2018) Features of the kinetics of mass transfer processes in the process of firing ceramic materials 4(41), doi: 10.26909/csl.4.2018.4

In article the technique of definition factors of carrying over weight of substance in the course of roasting ceramic materials which is based on exponent dependences of change mass bodies from time of its heating is offered.

The process of firing ceramic materials is associated with the transfer of heat and mass of matter. Therefore, for a more complete calculation of the heat treatment mode, it is necessary to know the conditions for the mass transfer in the product being calcined. The aim of the work is to determine the mass transfer coefficients of the substance in the process of firing ceramic materials.

The mass loss rate of the bound matter or the mass loss per unit time will be directly proportional to the average mass content of the body. On the other hand, the mass loss rate of the bound matter is numerically equal to the slope of the mass content kinetics curve.

Proposed in the work formulas can be applied when studying the process of mass transfer during firing of samples from different clays, as when firing clay samples of different diameters under the same conditions, they will differ in the mass content (mass loss rate of the bound substance) during heating and their final relative amount (mass content) of the lost mass will be the same.

Key words: kinetics of mass transfer processes, mass content.

\section{Особенности кинетики массообменных процессов в процессе обжига керамических материалов}

\author{
А.Н. Недбайло, А.Г. Чернышин \\ Государственное предприятие «Инженерный ичентр «Сушка»» Института технической теплофизики НАН \\ Украины, Киев, Украина
}

В статье предлагается методика определения коэффициентов переноса массы вещества в процессе обжига керамических материалов, которая основана на экспоненциальной зависимости изменения массосодержания тела от времени его нагрева.

Предложенные в работе формулы могут быть применены при изучении процесса массообмена при обжиге образцов из различных глин, так как при обжиге глиняных образцов различного диаметра при одинаковых условиях они между собой будут отличаться по изменению массосодержания (скорости массопотери связанного вещества) в процессе нагрева, а конечное их относительное количество (массосодержание) потерянной массы будет одинаковым. 


\section{Введение}

Процесс обжига керамических материалов связан с переносом теплоты и массы вещества. Следовательно, для более полного расчета режима термообработки необходимо знать условия перемещения массы в обжигаемом изделии.

Целью работы является определение коэффициентов переноса массы вещества в процессе обжига керамических материалов.

Коэффициенты переноса массы вещества (коэффициенты массообмена $\alpha_{\text {т }}$ и потенциалопрово-

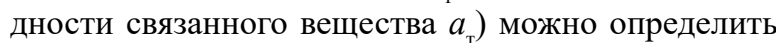
по кривой кинетики массосодержания, а также по кривым кинетики нагрева тела.

\section{Материалы и методы исследований}

В первом случае расчет производят по следующему методу. Известно, что в стадии регулярного режима кривая кинетики массосодержания (изменение массосодержания тела в зависимости от времени нагрева) описывается простой экспонентой $[1-3]$ :

$$
\frac{\bar{u}}{u}=A_{1} \exp \left(-\mu_{1}^{2} \frac{a_{m} \tau}{\mathrm{R}^{2}}\right)
$$

В этом случае скорость массопотери связанного вещества или убыль массы в единицу времени будет прямопропорциональна среднему массосодержанию тела, т.е. будет равна:

$$
-\frac{d u}{d \tau}=\frac{a_{m} \cdot \mu_{1}^{2}}{R_{2}} \bar{u},
$$

где $u$ - среднее массосодержание тела; $\tau$ - время; $\mu_{1}$ - первый корень характеристического уравнения; $R$ - определяющий размер тела.

Для модельных образцов (неограниченный цилиндр и пластина) $\mu_{1}$ может быть найдено по следующему приближенному уравнению:

$$
\frac{1}{\mu_{1}^{2}}=\frac{1}{\mu_{1 \infty}^{2}}+\frac{\Pi}{B i_{m}}
$$

где $\mathrm{Bi}_{m}=\alpha_{\mathrm{T}} \mathrm{R} / a_{\mathrm{T}}-$ массообменный критерий Био; П - постоянное число, для пластины равно 1 , для цилиндра $-1 / 2 ; \mu_{1 \infty}-$ первый корень характеристического уравнения при $\mathrm{Bi}_{m} \rightarrow \infty$ для образцов цилиндрической формы равен 2,405 , для пластины - 1,57.

Рассмотрим обжиг модельного образца в форме неограниченного цилиндра $(l \geq 2 d)$. В этом случае $\mu_{1}$, согласно уравнению (3), может быть найдено по следующему приближенному соотношению:

$$
\frac{1}{\mu_{1}^{2}}=\frac{1}{(2,405)^{2}}+\frac{a_{m}}{2 a_{m} R}
$$

С другой стороны, скорость массопотери связанного вещества численно равна тангенсу угла наклона кривой кинетики массосодержания:

$$
\operatorname{tg} \varphi=\frac{d u}{d \tau}=\frac{a_{m} \mu_{1}^{2}}{R^{2}} \bar{u}
$$

Из уравнений (1 - 3) получим:

$$
\frac{\bar{u}}{\operatorname{tg} \varphi}=\frac{R^{2}}{a_{m} \mu_{1}^{2}}=\frac{R^{2}}{(2,405)^{2} a_{m}}+\frac{R}{2 \alpha_{m}} .
$$

Пользуясь этой формулой, можно определить коэффициенты переноса массы вещества $a_{\mathrm{m}}$ и $\alpha_{\mathrm{m}}$, если известны значения $\operatorname{tg} \varphi$ и $\bar{u}$ для двух образцов различного радиуса, обожженных в одинаковых условиях.

\section{Результаты и их обсуждение}

Написав уравнение (6) для двух образцов различных радиусов, мы получаем систему двух уравнений, решение которых дает нам расчетные формулы соответственно для коэффициента потенциалопроводимости связанного вещества:

$$
a_{m}=\frac{R_{1} R_{2}\left(R_{1}+R_{2}\right)}{2,405^{2}\left(R_{1} \frac{\bar{u}_{2}}{\operatorname{tg} \varphi_{2}}-R_{2} \frac{\bar{u}_{2}}{\operatorname{tg} \varphi_{1}}\right)},
$$

и коэффициента массообмена:

$$
a_{m}=\frac{\operatorname{tg} \varphi_{1} \operatorname{tg} \varphi_{2} R_{1} R_{2}\left(R_{1}+R_{2}\right)}{2\left(R_{2}^{2} \bar{u}_{1} \operatorname{tg} \varphi_{2}-R_{1}^{2} \bar{u}_{2} \operatorname{tg} \varphi_{1}\right)} .
$$

Путем интегрирования уравнения (2) можно получить простое расчетное соотношение, из которого определяют коэффициент потенциалопроводимости связанного вещества и массообмена для случая, когда критерий $\mathrm{Bi}_{m} \rightarrow \infty$ :

$$
\int_{u_{1}}^{u_{2}} \frac{d u}{u}=\int_{\tau_{1}}^{\tau_{2}} \frac{a_{m} \mu_{1}^{2}}{R^{2}} d \tau
$$

или

$$
\ln u_{1}-\ln u_{2}=\frac{a^{\prime} \mu_{1}^{2}}{R^{2}}\left(\tau_{1}-\tau_{2}\right),
$$


и в окончательном виде

$$
\operatorname{tg} \varphi=\frac{2,3\left(\lg u_{1}-\lg u_{2}\right)}{\tau_{2}-\tau_{1}}
$$

учитывая, что при $\mathrm{Bi}_{\mathrm{m}} \rightarrow \infty$ для цилиндра $\mu_{1}=\mu_{1 \infty}=$ 2,405 , из уравнений (6) и (9) получаем формулы для определения коэффициентов переноса вещества $a_{m}$ и $\alpha_{m}$ :

$$
a_{m}=\frac{2,3\left(\lg u_{1}-\lg u_{2}\right) R^{2}}{\left(\tau_{2}-\tau_{1}\right)(2,405)^{2}}
$$

и

$$
\alpha_{m}=\frac{R}{2\left(\frac{\bar{u}\left(\tau_{2}-\tau_{1}\right)}{2,3\left(\lg u_{1}-\lg u_{2}\right) R^{2}}-\frac{R^{2}}{(2,405)^{2} a_{m}}\right)}
$$

Для определения $a_{\mathrm{m}}$ и $\alpha_{\mathrm{m}}$ по формулам (10) и (11) достаточно использовать кривые кинетики массообмена.

\section{Выводы}

Предложенные формулы (9) и (10) могут быть применены при количественной оценке интенсивности массообмена при обжиге образцов из различных глин. Так как при обжиге глиняных образцов различного диаметра при одинаковых условиях они между собой будут отличаться по изменению массосодержания (скорости массопотери связанного вещества) в процессе нагрева, а конечное их относительное количество (массосодержание) потерянной массы будет одинаковым.

\section{References}

1. Каспарян, Г.А. Описание процесса обжига глин при помощи обобщенных переменных. - Минск: Наука и техника. $-1996 .-82$ с.

2. Льков, А.В. Теория сушки. - М.: Энергия. 1968. $-471 \mathrm{c}$

3. Ралко, А.В. Экспериментальное исследование нестационарного тепло- и массобмена в процессе обжига. - ИФЖ. - 1956. - Т. 6. - С. 28 - 96. 